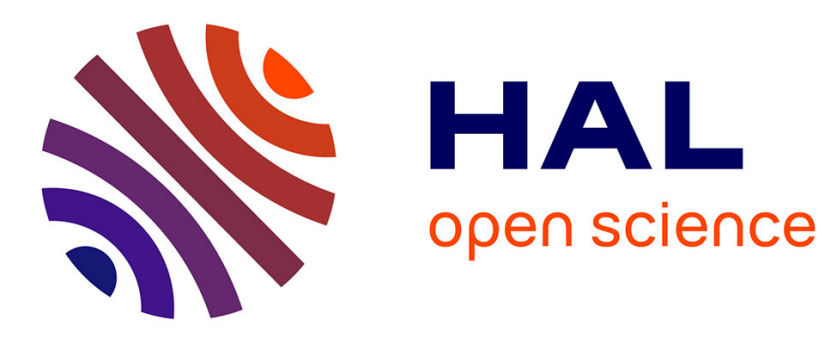

\title{
F-wave Amplitude Stability on Multiple Electrocardiogram Leads in Atrial Fibrillation
}

Marianna Meo, Antonio Hidalgo-Muñoz, Vicente Zarzoso, Olivier Meste, Gabriel Decebal Latcu, Nadir Saoudi

\section{- To cite this version:}

Marianna Meo, Antonio Hidalgo-Muñoz, Vicente Zarzoso, Olivier Meste, Gabriel Decebal Latcu, et al.. F-wave Amplitude Stability on Multiple Electrocardiogram Leads in Atrial Fibrillation. Computing in Cardiology, Sep 2015, Nice, France. hal-01217233

\section{HAL Id: hal-01217233 \\ https://hal.science/hal-01217233}

Submitted on 20 Oct 2015

HAL is a multi-disciplinary open access archive for the deposit and dissemination of scientific research documents, whether they are published or not. The documents may come from teaching and research institutions in France or abroad, or from public or private research centers.
L'archive ouverte pluridisciplinaire HAL, est destinée au dépôt et à la diffusion de documents scientifiques de niveau recherche, publiés ou non, émanant des établissements d'enseignement et de recherche français ou étrangers, des laboratoires publics ou privés. 


\title{
F-wave Amplitude Stability on Multiple Electrocardiogram Leads in Atrial Fibrillation
}

\author{
Marianna Meo ${ }^{1}$, Antonio R. Hidalgo-Muñoz ${ }^{2}$, Vicente Zarzoso², Olivier Meste ${ }^{2}$, Decebal G. Latcu ${ }^{3}$, \\ Nadir Saoudi ${ }^{3}$ \\ ${ }^{1}$ Brigham and Women's Hospital, Harvard Medical School, Boston, MA, USA \\ ${ }^{2}$ Laboratoire d'Informatique, Signaux et Systèmes de Sophia Antipolis (I3S), Université Nice Sophia \\ Antipolis, CNRS, France \\ ${ }^{3}$ Service de Cardiologie, Centre Hospitalier Princesse Grace, Monaco
}

\begin{abstract}
Fibrillatory wave (f-wave) amplitude correlates with left atrium (LA) size in certain electrocardiogram (ECG) leads and it is regarded as a predictor of ablation therapy outcome for atrial fibrillation (AF). This study aims at assessing the temporal stability of $f$-wave amplitude measures throughout the recording and determining the minimum signal length necessary to characterize them accurately in ECG leads.
\end{abstract}

In a set of standard ECGs acquired in 34 persistent $A F$ patients, we determined the minimum temporal window length $W$ such that the related amplitude value accurately correlated with that from the whole atrial activity $(A A)$ signal in leads I, II, $V_{1}-V_{6}$ (threshold Pearson's correlation coefficient $R=0.9$ ). Subsequently, we tested intrarecording correlation between amplitude values obtained in two distinct $W$-second AA signal excerpts. This procedure was performed both on the original AA signal and on its principal component analysis (PCA) rank-1 approximation.

The first experimental step yielded $W=5$ seconds. Amplitude intrarecording correlation was generally accurate in all leads for $W=5$ seconds $\left(R_{\min }=0.799, V_{1}\right.$; $\left.R_{\max }=0.999, V_{3}\right)$. Interestingly, PCA revealed that amplitude measures are more stable in proximity to $L A$ $\left(R\left(V_{1}\right)=0.975 ; R\left(V_{2}\right)=0.993 ; R\left(V_{3}\right)=0.989\right)$.

Our findings confirm the temporal stability of $f$-wave amplitude measures and their robustness to signal duration. Moreover, a preprocessing stage based on PCA improves the stability of this parameter in leads closer to LA.

\footnotetext{
This work is partly supported by the French National Research Agency under contract ANR-2010-JCJC-0303-01 PERSIST.

V. Zarzoso is a member of the Institut Universitaire de France.
}

\section{Introduction}

Atrial fibrillation (AF) is the most common sustained cardiac rhythm disturbance, increasing in prevalence with age [1]. It is associated with a wide range of potential complications and contributes significantly to population morbidity and mortality [2]. Standard electrocardiogram (ECG) is extensively employed in clinical practice for AF diagnosis and monitoring, since several ECG signal properties are significantly altered by this pathology. A relevant evidence is the absence of the $\mathrm{P}$ wave, which is replaced by irregular, rapid oscillations, the so-called fibrillatory waves (f-waves) [3,4], reflecting the disorganization of underlying atrial activity. According to their amplitude magnitude, they can be classified as fine $(<0.1 \mathrm{mV})$ or coarse f-waves $(>0.1 \mathrm{mV})[5,6]$. Furthermore, their amplitude is predictive of the outcome of catheter ablation (CA) of persistent forms of AF [7, 8], and a link with echocardiographic characteristics, in particular left atrial (LA) size, has been demonstrated is some studies [9-11].

Despite its clinical value, f-wave amplitude has been so far measured manually [7] or through semi-automatic methods [12]. As a consequence, its measure can be affected by variability due to interoperator differences and lack of measure repeatability. Furthermore, amplitude measurements are even more difficult to obtain in the presence of low signal-to-noise ratio and irregular patterns.

Meo et al. [8] have put forward a novel automatic method for f-wave amplitude computation. Peak-to-peak amplitude is evaluated by interpolating local extrema of the atrial activity (AA) signal, whose reduced-rank approximation is previously obtained by principal component analysis (PCA). The main goal of this study is to test the stability of f-wave amplitude measures within an ECG recording and their robustness to changes in AA signal pattern. This validation allows us to determine the minimum signal length required for accurate amplitude estimation. 


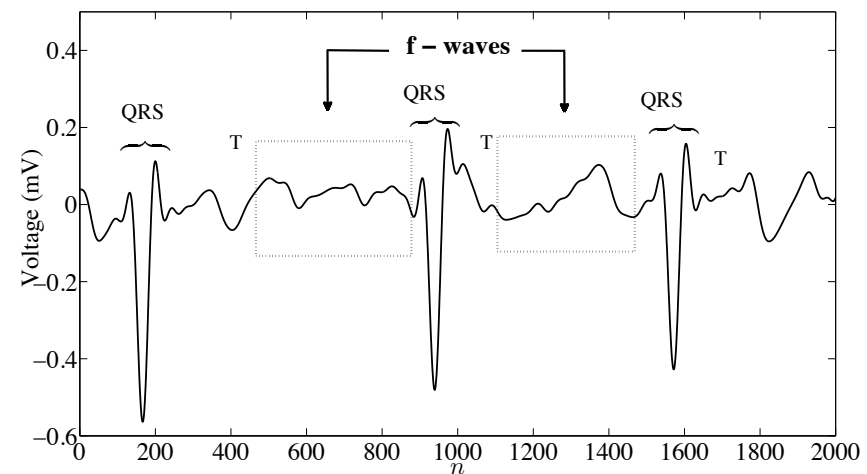

Figure 1. A representative example of an AF ECG recording and its fiducial points. TQ intervals are highlighted by boxes.

\section{Methods}

\subsection{Atrial activity signal extraction}

We examined a set of 12-lead ECG signals acquired right before $\mathrm{CA}$ in 34 patients affected by persistent $\mathrm{AF}$ and treated at the Cardiology Department, Princess Grace Hospital, Monaco. ECG signals were sampled at $977 \mathrm{~Hz}$. AA signal is obtained after QRST complex detection and removal from the input standard ECG recording. A signal excerpt in $\mathrm{V}_{1}$ is displayed in Fig. 1. Pan-Tompkins algorithm is applied to detect $\mathrm{R}$ wave time instants [13]. $\mathrm{Q}$ wave onset and $\mathrm{T}$ wave offset are estimated through the Woody's method [14]. Since AF dominant frequency is between 3 and $12 \mathrm{~Hz}$, a fourth-order zero-phase type II Chebyshev bandpass filter with $-3 \mathrm{~dB}$ attenuation between 0.5 and $30 \mathrm{~Hz}$ enables the suppression of noise caused by baseline wander, $50 \mathrm{~Hz}$ interference and other factors, e.g., myoelectric artifacts. TQ intervals are finally mean-corrected and concatenated, thus yielding the $(L \times N)$ AA signal as:

$$
\mathbf{Y}_{\mathrm{AA}}=\left[\mathbf{y}_{\mathrm{AA}(1)} \cdots \mathbf{y}_{\mathrm{AA}(\mathrm{N})}\right] \in \Re^{L \times N}
$$

where vector $\mathbf{y}_{\mathrm{AA}}(n)=\left[y_{1}(n), \ldots, y_{L}(n)\right]^{\mathrm{T}}$ is the multilead AA signal at sample index $n, L$ is the number of ECG leads considered, and $N$ the number of samples of the AA signal $y_{\ell}(n)$ for each lead $\ell=1,2, \ldots, L$. In this study, we considered a set of $L=8$ linearly independent ECG leads, namely, I, II, $\mathrm{V}_{1}-\mathrm{V}_{6}$.

\subsection{Multilead assessment of f-wave ampli- tude}

The single-lead algorithm for f-wave peak-to-peak amplitude computation is presented in [8]. Local minima and maxima of the atrial signal $y_{\ell}, \ell=1,2, \ldots, L$ are first detected. Subsequently, a lower and an upper envelope are estimated by the shape-preserving piecewise cubic Hermite interpolating polynomial (PCHIP) for each set of local extrema. The temporal average of $f$-wave amplitude is finally computed on the curve resulting from the difference between the two envelopes. In [8] this algorithm was applied to each ECG lead of the rank-1 PCA approximation to the input AA signal. In this study, to verify whether PCA actually improves amplitude estimation accuracy, we apply this algorithm to raw AA data $\mathbf{Y}_{\mathrm{AA}}$ as well.

\subsection{Accuracy evaluation of f-wave ampli- tude measurement}

The main goal of this study was to investigate whether f-wave amplitude characterization was affected by signal duration and determine the minimum AA signal duration $W$ necessary for accurate estimation of this parameter. Accordingly, we first evaluated the proportion of signal length preserved by TQ interval concatenation with respect to the length of the actual ECG recording in each AF patient. Secondly, we compared the amplitude value computed on a $W$-second temporal window (ranging between 1 and 10 s) with that obtained on the whole signal in each ECG lead introduced in Sec. 2.1. For the sake of consistency, this window was chosen at the beginning of the recording. Correlation was assessed by Pearson's correlation coefficient $R$; a threshold $R=0.9$ was set. Then, the optimal value of $W$ previously determined was employed to test intrarecording temporal stability of the f-wave amplitude measures by correlating amplitude values computed in two distinct $W$-second windows of the input AA signal, each positioned at the beginning and at the end of the recording, so as to avoid overlap influence. Additionally, this step was performed on the PCA rank-1 approximation to the AA signal with same duration $W$ as explained in [8], so as to verify whether atrial amplitude estimation can benefit from low-rank representations of the input signal.

\section{Results}

Duration of the AA signal $N_{A A}$ yielded by TQ interval concatenation was evaluated in the $\mathrm{AF}$ dataset under examination with respect to the length of the original ECG recording $N_{E C G}$ in terms of percentage ratio $\% N_{A A} / N_{E C G}$. Results are reported as mean \pm standard deviation $\left(\bar{N} \pm \sigma_{N}\right)$, minimum and maximum value ( $N_{M I N}$ and $N_{M A X}$, respectively) in Table 1.

In Fig. 2 the trend of Pearson's correlation coefficient $R$ is plotted as a function of the temporal window duration $W$, spanning the range from 1 to 10 seconds. Atrial amplitude is assessed both on AA signal and its PCA approximation. $R$ values quantify the correlation between the $\mathrm{f}$-wave amplitude value computed on the full signal and that evaluated on a $W$-second segment length at the beginning of 

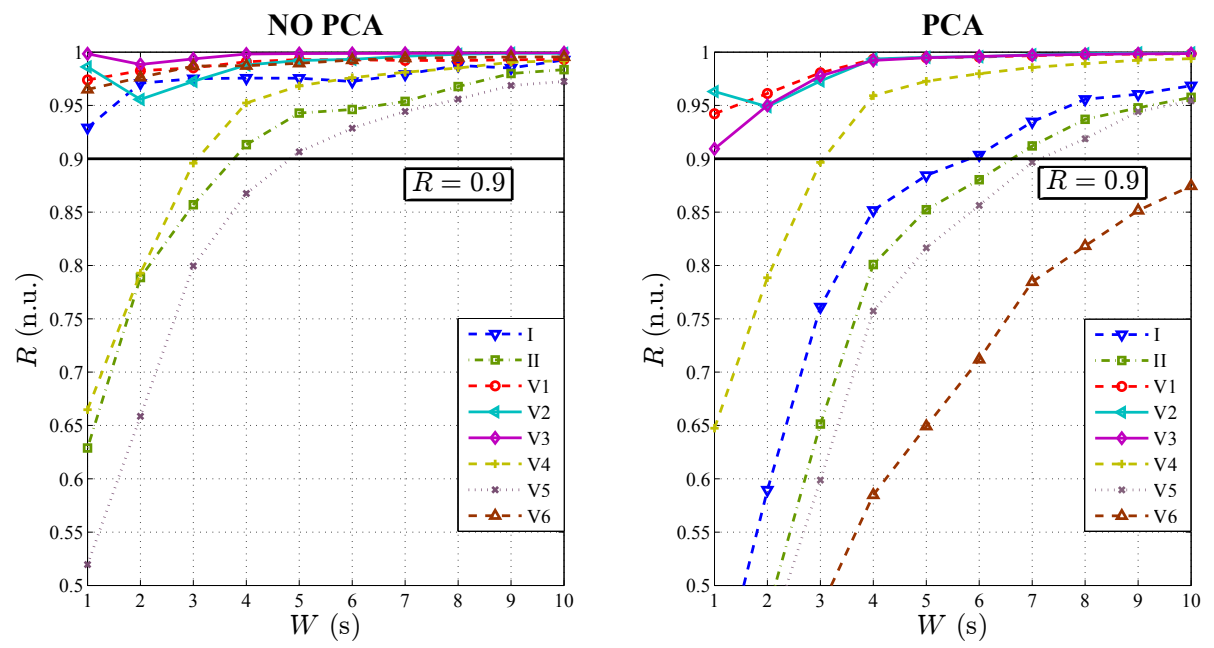

Figure 2. Pearson's correlation coefficient $R$ between amplitude values computed on the full recording and those from the $W$-second segments as a function of the window duration $W$ in ECG leads I, II, $\mathrm{V}_{1}-\mathrm{V}_{6}$.

Table 1. Average duration of the ECG signal in the AF database and percentage of AA signal preserved after ventricular activity suppression via TQ-segment concatenation.

\begin{tabular}{|c|c|c|c|}
\hline & $\bar{N} \pm \sigma_{N}$ & $N_{M I N}$ & $N_{M A X}$ \\
\hline$N_{E C G}[\mathrm{~s}]$ & $80.24 \pm 21.84$ & 48.98 & 139.94 \\
\hline$N_{A A}[\mathrm{~s}]$ & $41.66 \pm 12.12$ & 30.65 & 78.92 \\
\hline \hline$\% N_{A A} / N_{E C G}[\%]$ & $52.64 \pm 9.84$ & 35.93 & 80.19 \\
\hline
\end{tabular}

the AA signal itself. If we consider the raw atrial signal, a good trade-off between atrial amplitude estimation accuracy and minimal signal length can be reached by setting a threshold $R$ value equal to 0.9 , thus yielding $W=5$ seconds as the optimal window duration in every lead considered. Similar results are obtained in ECG leads $\mathrm{V}_{1}-\mathrm{V}_{4}$ only, if PCA preprocessing is applied to the AA signal.

In Table 2 Pearson's coefficient quantifies the intrarecording correlation between atrial amplitude values obtained in the initial and the terminal $W$-second signal portions. This evaluation is performed in each lead of the input AA signal and its minimum-rank PCA estimation.

\section{Discussion}

In [8] f-wave amplitude was assessed on the whole length of the available input AA signal. However, its temporal variations across the recording were not taken into account. Indeed, temporal average computation could mask potential sharp variations in the AA waveform, either intrinsic to the signal or due, for instance, to TQ interval concatenation. Furthermore, the influence of the signal duration on AA amplitude computation was not evaluated.
Table 2. Pearson's correlation between amplitude values estimated on $W$-second excerpts extracted at the beginning and at the end of the input signal in ECG leads I, II, $\mathrm{V}_{1}-\mathrm{V}_{6}$ ( $W=5$ seconds). F-wave amplitude is computed both on the raw AA signal and its rank-1 PCA approximation.

\begin{tabular}{|c|c|c|}
\hline & No PCA & PCA \\
\hline$R(I)$ & 0.861 & 0.892 \\
\hline$R(I I)$ & 0.916 & 0.841 \\
\hline$R\left(V_{1}\right)$ & 0.799 & 0.975 \\
\hline$R\left(V_{2}\right)$ & 0.985 & 0.993 \\
\hline$R\left(V_{3}\right)$ & 0.999 & 0.989 \\
\hline$R\left(V_{4}\right)$ & 0.957 & 0.961 \\
\hline$R\left(V_{5}\right)$ & 0.864 & 0.798 \\
\hline$R\left(V_{6}\right)$ & 0.986 & 0.576 \\
\hline
\end{tabular}

Results in Table 1 show that on average approximately half of the input ECG signal is discarded after QRST complex removal, considering that the span of ECG duration values in our AF database is quite broad (from 30 seconds to approximately 2 minutes). Our findings show that amplitude quantification is more stable in ECG leads closer to $\mathrm{V}_{1}$. Indeed, in leads $\mathrm{I}, \mathrm{V}_{1}, \mathrm{~V}_{2}, \mathrm{~V}_{3}$ and $\mathrm{V}_{6}$, coefficient $R$ was barely affected by signal window duration, and correlation was overall high $(R>0.9)$, whereas in the remaining ECG leads too short temporal segments seem to affect amplitude quantification, as proved by the poor correlation with the full signal counterpart ( $R$ close to 0.5 ) for short window lengths $W$ in Fig. 2. This correlation test demonstrates that we can accurately estimate f-wave amplitude in quite short AA segments ( $W=5$ seconds), therefore giving the possibility to consider even ECGs of limited duration (much less than one minute), which are easier to ac- 
quire in clinical routine, or discard signal portions affected by noise or other artifacts without losing information. Correlation was slightly weaker when PCA was applied, since we should retain at least 7 seconds of the AA signal in most of the leads for the same threshold $R=0.9$.

Robustness of f-wave amplitude measures estimated by the method previously illustrated is further corroborated by correlation results presented in Table 2 . Their temporal stability across the ECG recording is confirmed by high $R$ values in all the ECG leads when raw AA data are examined.The minimum Pearson's coefficient value was found in $\mathrm{V}_{1}\left(R_{\min }=0.799\right)$, whereas lead $\mathrm{V}_{3}$ exhibited the highest correlation $\left(R_{\max }=0.999\right)$. On the other hand, correlation results appear to be more variable when AA signal is preprocessed by PCA. This outcome may be explained by the PCA filtering effect, which condenses the most significant information in one component, while removing redundant and/or polluting elements. Interestingly, more stable amplitude measures were provided by PCA in proximity to $\mathrm{LA}$, such as in $\mathrm{V}_{1}$ and $\mathrm{V}_{3}$. By contrast, ECG leads further from this site exhibited poor correlation, such as in $V_{5}$ and $\mathrm{V}_{6}$. A possible explanation may come from LA structural remodeling during $\mathrm{AF}$, whose hallmark is dilation, altering electrical activity from the single myocyte scale up to the whole tissue level [15]. Such alterations can also be detected on ECG signals, and the orientation of certain leads may better reflect the pathological conditions of this site.

\section{Conclusions}

Our study demonstrates f-wave amplitude can be accurately estimated even in very short ECG recordings without losing meaningful information. This may be helpful in certain situations when a signal portion must be discarded (e.g., for noise or acquisition issues) or when a very specific episode is examined. Moreover, atrial amplitude measures prove to be stable throughout the recording and are not substantially affected by signal temporal variations or TQ-segment concatenation. Finally, PCA preprocessing of the AA signal improves amplitude estimation in ECG leads closer to LA, and may reflect alterations due to electrical remodeling.

\section{References}

[1] January C, Wann L, Alpert J, et al. 2014 AHA/ACC/HRS Guideline for the management of patients with atrial fibrillation: Executive summary: A report of the American College of Cardiology/American Heart Association Task Force on Practice Guideline and Heart Rhythm Society. J Am Coll Cardiol 2014;64(21):2246-2280.

[2] Nattel S, Burstein B, Dobrev D. Atrial remodeling and atrial fibrillation : Mechanisms and implications. Circ Arrhythmia Electrophysiol 2008;5:62-73.
[3] Thurmann M, Janney JG. The diagnostic importance of fibrillatory wave size. Circulation 1962;25:991-994.

[4] Xi Q, Sahakian AV, Ng J, Swiryn S. Atrial fibrillatory wave characteristics on surface electrogram: ECG to ECG repeatability over twenty-four hours in clinically stable patients. J Cardiovasc Electrophysiol 2004;15(8):911-917.

[5] Peter R, Morris J, McIntosh H. Relationship of fibrillatory waves and $\mathrm{P}$ waves in the electrocardiogram. Circulation 1966;33:599-606.

[6] Leier C, Schaal S. Biatrial electrograms during coarse atrial fibrillation and flutter-fibrillation. Am Heart J 1980; 99:331-341.

[7] Nault I, Lellouche N, Matsuo S, at al. Clinical value of fibrillatory wave amplitude on surface ecg in patients with persistent atrial fibrillation. J Interv Card Electrophysiol 2009;26:11-19.

[8] Meo M, Zarzoso V, Meste O, Latcu DG, Saoudi N. Spatial variability of the 12-lead surface ECG as a tool for noninvasive prediction of catheter ablation outcome in persistent atrial fibrillation. IEEE Trans Biomed Eng Jan. 2013; 60(1):20-27.

[9] Aysha MH, Hassan AS. Diagnostic importance of fibrillatory wave amplitude: a clue to echocardiografic left atrial size and etiology of atrial fibrillation. J Electrocardiol 1988; 21(3):247-251.

[10] Yamamoto S, Suwa M, Ito T, et al. Comparison of frequency of thromboembolic events and echocardographic findings in patients with chronic nonvalvular atrial fibrillation and coarse versus fine electrocardiographic fibrillatory waves. Am J Cardiol 2005;96:408-411.

[11] Mutlu B, Karabulut M, Eroglu E, et al. Fibrillatory wave amplitude as a marker of left atrial and left atrial appendage function, and a predictor of thromboembolic risk in patients with rheumatic mitral stenosis. Int J Cardiol 2003;91:179_ 186.

[12] Hoppe B, Kahn AM, Feld G, Hassankhani A, Narayan SM. Separating atrial flutter from atrial fibrillation with apparent electrocardiographic organization using dominant and narrow f-wave spectra. J Am Coll Cardiol 2005;46(11):2079_ 2087.

[13] Pan J, Tompkins WJ. A real-time QRS detection algorithm. IEEE Trans Biomed Eng 1985;3(3):230-236.

[14] Cabasson A, Meste O. Time delay estimation: A new insight into the Woody's method. IEEE Signal Proc Let 2008; 15:573-576.

[15] Casaclang-Verzosa G, Gersh BJ, Tsang TSM. Structural and functional remodeling of the left atrium: clinical and therapeutic implications for atrial fibrillation. J Am Coll Cardiol 2008;51(1):1-11.

Address for correspondence:

Marianna Meo

Brigham and Women's Hospital, Harvard Medical School

20 Shattuck Street, 02119 Boston, MA, USA

mariannameo@gmail.com 